\title{
Magnetic Massive Stars
}

\author{
Rich Townsend ${ }^{1}$, David H. Cohen ${ }^{2}$, Luc Dessart ${ }^{3}$, Swetlana Hubrig ${ }^{4}$, \\ Yaël Nazé ${ }^{5}$, Véronique Petit ${ }^{6}$, Asif ud-Doula ${ }^{1}$ and Nolan R. Walborn ${ }^{7}$ \\ ${ }^{1}$ Bartol Research Institute, University of Delaware, Newark, DE 19716, USA \\ ${ }^{2}$ Swarthmore College, Department of Physics and Astronomy, 500 College Ave., Swarthmore \\ College, Swarthmore, PA 19081, USA \\ ${ }^{3}$ Department of Astronomy and Steward Observatory, University of Arizona, Tucson, AZ \\ 85721, USA \\ ${ }^{4}$ European Southern Observatory, Casilla 19001, Santiago, Chile \\ ${ }^{5}$ Institut d'Astrophysique et de Géophysique, Université de Liège, Bât. B5c, Allée du VI Aût \\ 17, B-4000 Liège, Belgium \\ ${ }^{6}$ Département de Physique, de Génie Physique et d'Optique and Observatoire du mont \\ Mégantic, Université Laval, Québec, QC G1K 7P4, Canada \\ ${ }^{7}$ Space Telescope Science Institute, 3700 San Martin Drive, Baltimore, MD 21218, USA
}

\begin{abstract}
Magnetic fields are unexpected in massive stars, due to the absence of a sub-surface convective dynamo. However, advances in instrumentation over the past three decades have led to their detection in a small but growing subset of these stars. Moreover, complementary theoretical developments have highlighted their potentially significant influence over the structure, evolution and circumstellar environments of massive stars. Here, we summarize a special session convened prior to the main conference, focused on presenting recent developments in the study of massivestar magnetic fields.
\end{abstract}

Keywords. stars: early-type, magnetic fields, stars: winds, stars: mass-loss, hydrodynamics, MHD, techniques: spectroscopic, techniques: polarimetric, X-rays: stars, supernovae: general

\section{Optical, Ultraviolet, and X-Ray Spectral Morphology of Hot Magnetic Stars}

Nolan R. Walborn

It is noteworthy that the four hottest massive magnetic stars were all isolated as peculiar from their spectral morphology and/or variability prior to the field detections. They are the mid-O stars $\theta^{1}$ Orionis $\mathrm{C}$ and HD 191612, and the early-B stars $\tau$ Scorpii and $\xi^{1}$ Canis Majoris. These peculiarities extend to the optical, UV, and X-ray domains, in comparison with the corresponding normal spectral sequences. Indeed, it is essential to recognize and isolate the peculiar objects, which otherwise obscure the normal trends. The existence of the latter in X-rays is a surprising new development, emerging from purely morphological analysis of Chandra data as a function of the optical spectral types, just as the UV wind-profile systematics did from IUE data during the early 1980's. Figure 1 shows a normal X-ray spectral-type sequence; the progression of the strongest lines toward longer wavelengths with advancing spectral type, and the correlated H-like/Helike line ratios of $\mathrm{Si}, \mathrm{Ne}$, and $\mathrm{O}$, are readily seen. The Ne behavior is most useful, since the ratio reverses through this domain (note interference from an Fe XVII line at the later types). In contrast, Figure 2 shows the drastic departures of some magnetic and rapidly rotating stars from the normal trends. (Figures are courtesy of Wayne Waldron.) 


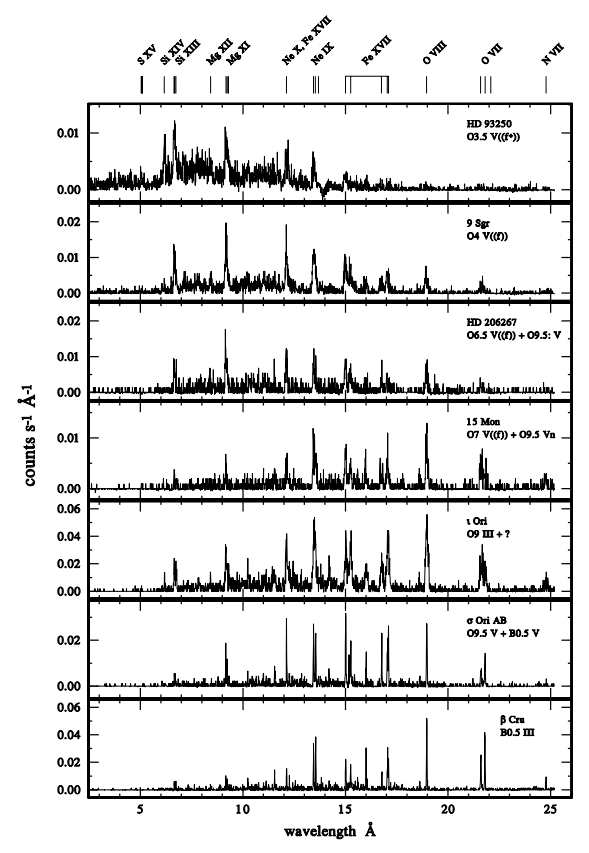

Figure 1.

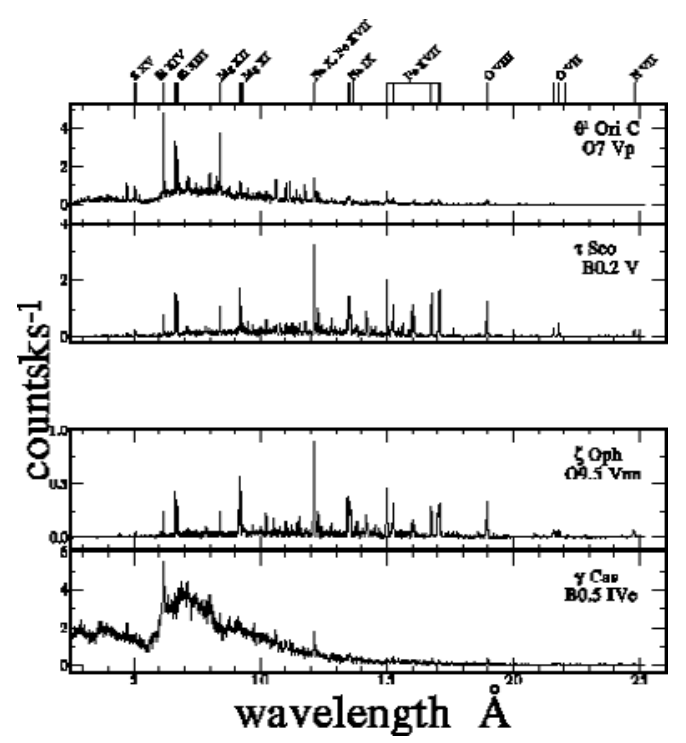

Figure 2.

These results have two important implications: (1) other hot, massive stars with unexplained spectral peculiarities or variations are strong magnetic candidates that should be observed for fields (HD 108, HD 36879, HD 148937, $\theta$ Car, 15 Mon); and (2) the rarity of these phenomena indicates that the fields are likely primordial, rather than generated by the intrinsic stellar structure and evolution. (Acknowledgements: NASA/FUSE grant NNG06G179G).

\section{The Mysterious Of?p Class and the Magnetic O-Star $\theta^{1}$ Ori C Yaël Nazé}

In our Galaxy, there are only 3 stars in the Of?p category: HD 108, HD 148937 and HD 191612. Their peculiar properties (varying line profiles, X-ray over-luminosity, and photometric changes) were recently discovered. A magnetic field was even detected for HD 191612, leading to the suggestion that this object was a magnetic oblique rotator, similar to $\theta^{1}$ Ori $\mathrm{C}$ but older. We review here the physical properties of these objects and re-examine the evidence leading to that conclusion.

- Spectral types: That of $\theta^{1}$ Ori $\mathrm{C}$ is still uncertain (O5.5-O7V), but it is obvious that the star does not share the defining characteristics of the Of?p category, i.e. strong

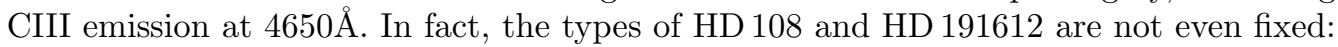
due to contamination by varying emission, they alternate between early Of?p (O4-5.5) with strong CIII emission and late $\mathrm{O}(\mathrm{O} 8-8.5)$ with a much weaker CIII emission.

- Variability: H and HeII 4686 are variable for all objects of our sample, but the CIII and HeI lines also vary strongly for HD 108 and HD 191612. Note that the changes in HD 148937 are of very weak amplitude.

- Periodicity: The line profile changes are periodic with a timescale of $15.4 \mathrm{~d}$ for $\theta^{1}$ Ori C, 537.6d for HD 191612, 55yrs for HD 108 and possibly 7d for HD 148937. 
- Binarity: Both $\theta^{1}$ Ori C and HD 191612 are eccentric binaries with $M_{1} / M_{2} \sim 0.5$. The orbital period is much longer than that of the line profile changes: $10.9 y$ rs for the former and 1542d for the latter. The binary status of HD 108 and HD 148937 is not yet fully ascertained: no clear evidence for orbital motion was detected, but very long-term and/or low-amplitude velocity variations can not be excluded.

- Physical parameters: The Fourier method for determining rotation rate shows that all of these objects present low $v \sin (i)$. Atmosphere model fits yields similar values of the luminosity and temperature for HD 108, HD 191612 and $\theta^{1}$ Ori C: these stars appear very close to each other in the HR diagram while HD 148937 is twice more luminous.

- Other characteristics: A magnetic field was clearly detected for $\theta^{1}$ Ori $\mathrm{C}$ and HD 191612, but observations are needed to check if the other two Of?p objects are magnetic. HD 148937 is surrounded by a nebula, thought to have been ejected during a LBV-like event; the star and its nebula are both clearly enriched in nitrogen, suggesting that HD 148937 might be more evolved than the other 3 stars.

- Photometry: Both HD 108 and HD 191612 appear brighter when the emission lines are stronger and the spectral type earlier. No large variation of the photometry of HD 148937 was found; photometric changes in $\theta^{1}$ Ori $\mathrm{C}$ have been proposed to explain the small variations of the EW of the photospheric lines, but have not been confirmed yet.

- X-ray properties: At high energies, $\theta^{1}$ Ori C displays a large overluminosity, a very hard spectrum (dominated by a temperature of $\sim 3 \mathrm{keV}$ ), phase-locked variations (simultaneous brightening of the X-ray and visible emissions), and very narrow lines. The X-ray spectrum of the three Of?p is clearly different: though a large overluminosity is present, their spectrum is soft (dominated by a temperature of $\sim 0.3 \mathrm{keV}$ ) and displays rather broad lines. For HD 191612, flux variations are also present, but their phase-locked nature is uncertain.

It is now clear that $\theta^{1}$ Ori $\mathrm{C}$ and the Of?p stars share many similarities. However, there are also crucial differences that should not be discarded, especially in the X-ray domain. It seems thus premature to simply identify these objects with $\theta^{1}$ Ori C. Most probably, what is observed is a combination of effects due to the magnetic field with a completely different phenomenon (still unknown). (Acknowledgement: FRS-FNRS for grant and support).

\section{Observations of Magnetic Fields of Massive Stars with FORS 1 at the VLT}

Swetlana Hubrig

Our recent studies using FORS 1 in spectropolarimetric mode included observations of magnetic fields in a sample of massive stars with spectral types earlier than B2. The excellent potential of FORS 1 for measuring magnetic fields in massive stars was demonstrated by observations of the mean longitudinal magnetic field in $\theta^{1}$ Ori C in 2007 (Hubrig et al. 2008). This star was the first O-type star with a detected weak magnetic field varying with a rotation period of 15.4 days. The FORS 1 measurements show a sinusoidal curve in spite of the phase gap between 0.60 and 0.88 . However, our observations determined a magnetic geometry distinct from the one deduced by Wade et al. (2006). The maxima and minima of the measured longitudinal field as well as the phases of the field extrema appeared to be significantly different. Assuming an inclination of the rotation axis to the line-of-sight of $i=45^{\circ}$, our modeling of the longitudinal field variation constrains the 
dipole magnetic field geometry of $\theta^{1}$ Ori $\mathrm{C}$ to $B_{\mathrm{d}} \approx 900 \mathrm{G}$ and $\beta \approx 80^{\circ}$, where $B_{\mathrm{d}}$ is the dipole intensity and $\beta$ is the obliquity angle.

A longitudinal magnetic field at a level larger than $3 \sigma\left(\left\langle B_{z}\right\rangle=-115 \pm 37 \mathrm{G}\right)$ has been detected by us in another O-type star, HD 155806, with a spectral type O7.5IIIe, which is currently the third O-type star with a diagnosed magnetic field. Clear variations of SiIV, HeI and other lines have been detected in FEROS and UVES spectra retrieved from the ESO archive (Hubrig et al. in preparation).

Our search of magnetic fields in a sample of 13 Be stars revealed the presence of weak magnetic fields in two stars, HD 56014 and HD 148184 (Hubrig et al. 2007a). For two other stars in the studied sample, HD 58011 and HD 11735, we detected distinctive circular polarization signatures in the Stokes V spectra of the CaII H\&K lines. The profiles of these Ca lines in the FORS 1 spectra taken in integral light are deeper than predicted by synthetic spectra. Additional high signal-to-noise spectroscopic observations are needed to study the Ca line profiles to be able to decide whether they are formed in circumstellar disks around these stars.

Finally, we report the discovery of weak magnetic fields in a few $\beta$ Cephei stars and in about 20 slowly pulsating B (SPB) stars (Hubrig et al. 2006; Hubrig et al. in preparation) implying that massive pulsating stars can no longer be considered as classes of nonmagnetic pulsators. However, the effect of the fields on the oscillation properties remains to be studied.

Our spectropolarimetric studies using FORS 1 at the VLT demonstrate that magnetic fields are indeed present in massive stars. For the case of magnetic fields in massive stars that are weaker in strength and likely more complex in their geometry than classical magnetic Ap and Bp stars, progress in their study may potentially come from detailed studies of polarized line profiles. At present, it is not obvious to what extent magnetic fields can be directly discovered in circumstellar material. Previous detections of magnetic fields in circumstellar material include a detection of magnetic fields in the circumstellar disk of FU Ori (Donati et al. 2005) and in circumstellar Ca lines of Herbig stars (Hubrig et al. 2006; Hubrig et al. 2007). However, modeling diagnostics of magnetic fields in these environments are still under development (Ignace \& Gayley 2007).

\section{X-ray Emission and the Incidence of Magnetic Fields in Massive Stars of the Orion Nebula Cluster}

Véronique Petit

Magnetic fields have been frequently proposed as a likely source of variability and confinement of the winds of massive stars. Recently, Stelzer et al. (2005) found significant X-ray emission from all massive stars in the Orion Nebula Cluster (ONC). Possibly periodic rotational modulation in $\mathrm{X}$-rays and other indicators suggested that there might be many magnetic B- and O-type stars in this star-forming region.

Magnetic fields can be directly detected in stellar atmospheres by the means of the Zeeman effect. If the field is strong enough, and the spectral lines narrow enough, one can directly see the Zeeman splitting of lines in the intensity spectrum. However, if the field is weaker, and the lines broadened either intrinsically or by fast rotation, the splitting is much more difficult to detect, even at high spectral resolution. In that case, the most effective way to detect magnetic fields is to look for circular polarization signatures across photospheric spectral lines, generated by the longitudinal Zeeman effect. During the past decade, a powerful multi-line analysis procedure called 'Least Squares Deconvolution' (LSD; Donati et al. 1997) has been developed and applied for extracting a mean Stokes 
$\mathrm{V}$ profile from a stellar spectrum, simultaneously exploiting the signal contained in all the lines present. This allows for significantly improved sensitivity to magnetic fields, as it substantially increases the signal-to-noise ratio.

Notwithstanding the gains provided by these sophisticated tools, magnetic fields in hotter OB stars remain a challenge to detect. The few photospheric lines present in the optical spectrum and the large intrinsic width of these lines, compounded by the usual rapid rotation of these stars, require large-bandwidth and high signal-to-noise ratio observation to start with, even using LSD. The advent of a new generation of spectropolarimeters such as ESPsDOnS at the Canada-France-Hawaii Telescope (CFHT) and its twin NARVAL at the Télescope Bernard-Lyot (TBL) now allows a new level of investigation of magnetic fields in massive OB stars. ESPaDOnS consists of a polarimetric module located at the Cassegrain focus of the CFHT, linked by optical fibers to the high-resolution echelle spectrometer. A resolution of 65,000 for a spectral range covering $360 \mathrm{~nm}$ to $1 \mu \mathrm{m}$ can be achieved in a single observation. The high spectral resolution enables the resolution of the Zeeman signature across essentially all individual spectral lines, which provides a qualitative advantage over lower resolution instruments.

We have carried out sensitive ESPaDOnS observations to search for direct evidence of magnetic fields in the massive stars of the ONC. We used the statistical test described by Donati et al. (1997) to diagnose the presence of a signal in LSD mean Stokes V profiles. A signal is unambiguously detected whenever the associated detection probability is larger than 99.999 per cent (corresponding to a false alarm probability smaller than $\left.10^{-5}\right)$, and when no signature is detected in the associated diagnostic null (N) spectrum. The associated longitudinal field can be inferred from the Stokes I and V profiles. It is important to note that the longitudinal field is not the primary diagnostic for inferring the presence of a magnetic filed, because a magnetic configuration with a null longitudinal component still usually produces a non-zero Stokes V signature.

We report the detection of two new massive magnetic stars in the Orion Nebula Cluster: Par 1772 (HD 36982) and NU Ori (HD 37061), for which the estimated dipole polar field strengths, with $1 \sigma$ error bars, are $1150_{-200}^{+320} \mathrm{G}$ and $650_{-170}^{+220} \mathrm{G}$ respectively. We also obtain dipole field upper limits for the remaining stars with a state-of-the-art Bayesian analysis, resulting in a precise magnetic characterization of all ONC massive stars. This allows us to explore for the first time the connections between fields, winds and X-rays in a complete, co-eval and co-environmental sample of massive stars. These remarkable results bring forth new challenges for understanding the processes leading to X-ray emission in massive stars. We also expect to provide unique data regarding the incidence of magnetic fields in massive stars with which to confront models of magnetic field origin in neutron stars and magnetars, such as that proposed by Ferrario \& Wickramasinghe (2006).

\section{X-rays from Magnetically Channeled Winds of OB Stars David Cohen}

OB stars with strong radiation-driven stellar winds and large-scale magnetic fields generate strong and hard X-ray emission via the Magnetically Channeled Wind Shock (MCWS) mechanism (Shore \& Brown 1990; Babel \& Montmerle 1997; ud-Doula \& Owocki 2002). There are four separate X-ray diagnostics that confirm the MCWS scenario for the young, magnetized $\mathrm{O}$ star that illuminates the Orion Nebula, $\theta^{1}$ Ori $\mathrm{C}$, and constrain the physical properties of its X-ray emitting magnetosphere:

1. High $\mathrm{X}$-ray temperatures, determined from thermal spectral model fitting. The differential emission measure of $\theta^{1}$ Ori $\mathrm{C}$ peaks at temperatures above $10 \mathrm{MK}$, which 
is in contrast to the few million $\mathrm{K}$ peak temperatures in mature, unmagnetized $\mathrm{O}$ stars (Wojdowski \& Schulz 2005), and which is well reproduced by MHD simulations of the MCWS mechanism (Gagné et al. 2005).

2. Relatively narrow X-ray emission lines. The X-ray emitting plasma in the MCWS scenario is predominantly in the closed magnetic field regions and thus the plasma velocity is relatively low and the associated Doppler line broadening is modest. This is seen in the MHD simulations and confirmed by the Chandra grating observations.

3. The rotational modulation of the X-ray emission is consistent with part of the magnetosphere being eclipsed near phase 0.5 , when the viewing orientation is magnetic equator-on. The depth of the eclipse provides information about the location of the Xray emitting plasma (deeper eclipses imply more plasma close to the star). The observed eclipse depth for $\theta^{1}$ Ori $\mathrm{C}$ implies that the bulk of the plasma is within a stellar radius of the photosphere. This is a somewhat closer than the MHD simulations predict (e.g. Gagné et al. 2005).

4. The ratio of the forbidden to intercombination line strengths in helium-like ions also puts a constraint on the location of the X-ray emitting plasma, via the sensitivity of these line ratios to the local UV mean intensity. The closer the hot plasma is to the photosphere, the stronger the UV photoexcitation of electrons from the upper level of the forbidden line to the upper level of the intercombination line, and the smaller the $f / i$ ratio. This is demonstrated in Fig. 3 for the $\mathrm{Mg}$ XI complex in the co-added (over four observations) Chandra grating spectrum of $\theta^{1}$ Ori C, where we see that the very weak forbidden line requires a plasma location below $r \approx 2 \mathrm{R}_{*}$.

\section{MHD Simulation of Magnetic Channeling and Spindown in Rotating Hot-Star Winds}

Asif ud-Doula

Building upon our previous MHD simulation studies of magnetic channeling in radiatively driven stellar winds, this talk examines the dynamical effects of stellar rotation in the 2-D axisymmetric case of an aligned dipole surface field. In addition to the magnetic confinement parameter $\eta_{*} \equiv B_{\text {eq }}^{2} R_{*}^{2} / \dot{M} v_{\infty}$ introduced in ud-Doula \& Owocki (2002), we now add a rotational parameter $W \equiv V_{\text {rot }} / V_{\text {orb }}$ (the ratio of the equatorial surface rotation speed to orbital speed), examining specifically models with moderately strong rotation $\mathrm{W}=0.25$ and 0.5 , and comparing these to analogous nonrotating cases. Defining the associated Alfvén radius $R_{\mathrm{A}} \approx \eta_{*}^{1 / 4} R_{*}$ and Kepler corotation radius $R_{\mathrm{K}} \approx W^{-2 / 3} R_{*}$, we find rotation effects are weak for models with $R_{\mathrm{A}}<R_{\mathrm{K}}$, but can be substantial and even dominant for models with $R_{\mathrm{A}}>R_{\mathrm{K}}$. In particular, by extending our simulations to magnetic confinement parameters (up to $\left.\eta_{*}=1000\right)$ that are well above those $\left(\eta_{*}=10\right)$ considered in ud-Doula \& Owocki (2002), we are able to study cases with $R_{\mathrm{A}} \gg R_{\mathrm{K}}$; we find that these do indeed show clear formation of the rigid-body disk predicted in previous analytic models (Townsend and Owocki 2005), with however a rather complex, dynamic behavior characterized by both episodes of downward infall and outward breakout that limit the buildup of disk mass. Overall, the results provide an intriguing glimpse into the complex interplay between rotation and magnetic confinement, and form the basis for a full MHD description of the rigid-body disks expected in strongly magnetic Bp stars like $\sigma$ Ori E.

This simulation study also allows us to examine the role of such rotation-aligned dipole filed in enhancing the net loss of angular momentum from the wind. Compared to the 1D analytic model derived by Weber and Davis (1967; WD) for the effect of a monopole 

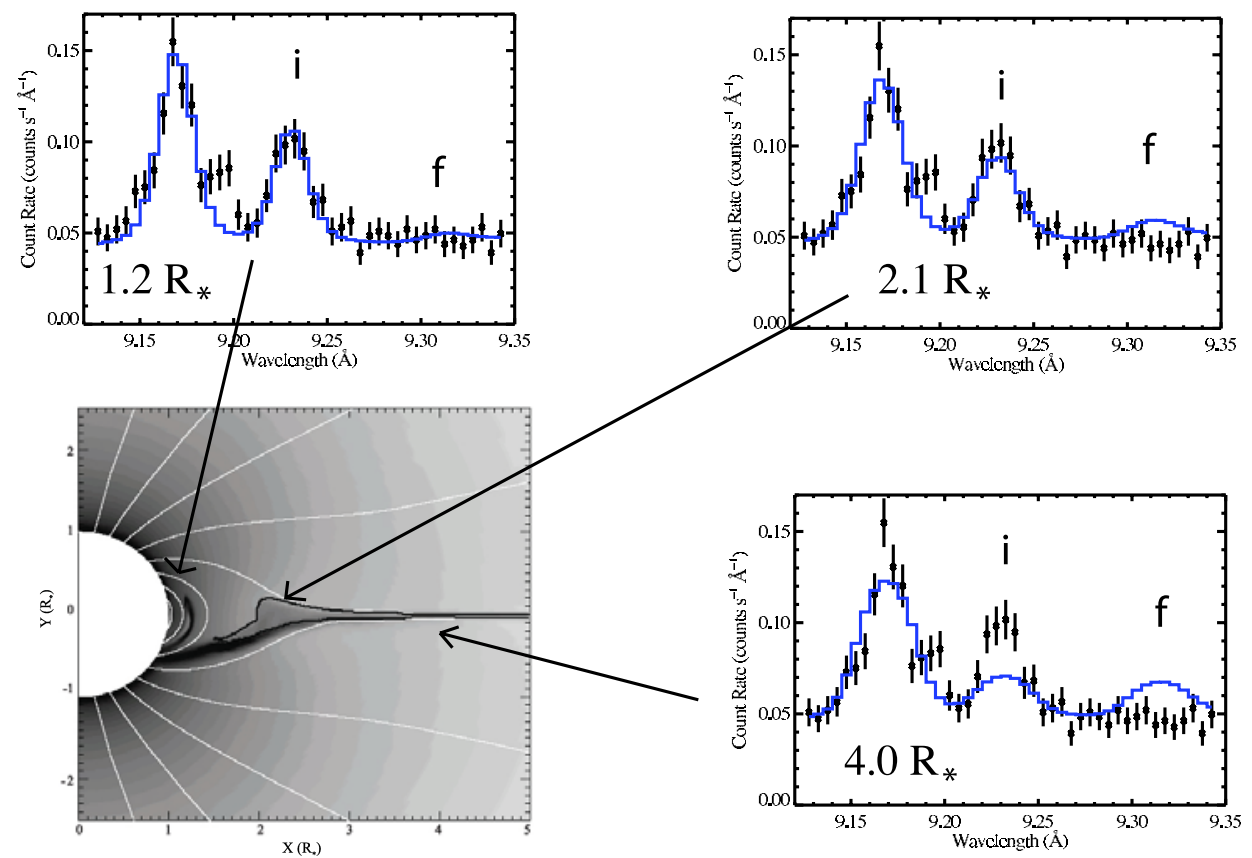

Figure 3. A snapshot from a 2-D MHD simulation of $\theta^{1}$ Ori C, taken from Gagné et al. (2005), showing emission measure in grayscale, magnetic field lines as white contours, and with a thick, black contour enclosing plasma with temperature above $10^{6} \mathrm{~K}$ (lower left). The three other panels show the Chandra spectrum in the vicinity of the helium-like $\mathrm{Mg}$ XI complex, with a model of the resonance, intercombination $(i)$, and forbidden $(f)$ lines overplotted. The relative strengths of the $f$ and $i$ lines are different in each of the three panels, as the three models were calculated assuming a source location of $1.2 \mathrm{R}_{*}, 2.1 \mathrm{R}_{*}$, and $4.0 \mathrm{R}_{*}$, respectively, starting at the top left and moving clockwise. The arrows indicate the approximate location in each case. The intermediate case $-r=2.1 \mathrm{R}_{*}$, which seems to agree with the MHD simulation - is marginally consistent with the data ( $68 \%$ confidence limit).

field on the equatorial plane outflow of the gas-pressure-driven solar, our 2D simulations of rotation-aligned dipole in a line-driven stellar wind show much more complex, timedependent flows; but the overall scaling for the net loss of angular momentum still can be cast in terms similar to the classic WD form, $\dot{J} \approx-(2 / 3) \dot{M} \Omega R_{A}^{2}$, where $\Omega$ the stellar rotation frequency. As in the WD model, only ca. $20 \%$ of this angular momentum is carried by wind material itself, with the bulk of the loss instead carried by the field Poynting stress. However, while the WD monopole model implies a scaling $R_{\mathrm{A}} \sim \eta_{*}^{1 / 2} R_{*}$, our more realistic dipole simulation models give a smaller Alfvén radius $R_{\mathrm{A}} \sim \eta_{*}^{1 / 4} R_{*}$. The associated stellar rotation spindown time then has the scaling $t_{J} \equiv J / \dot{J} \approx 0.05 t_{M} / \sqrt{\eta_{*}}$, where $t_{M} \equiv M / \dot{M}$ is the characteristic mass loss time. For strong magnetic rotators like $\sigma$ Ori E, the expected spindown time is of order a million years or shorter.

\section{Rigid-Field Hydrodynamics Models for the Magnetospheres of Strongly Magnetic Massive Stars}

Rich Townsend

Among the differing classes of magnetic massive star, the 'He-strong' stars - characterized by elevated and spatially inhomogeneous photospheric helium abundances — are 
the most challenging to model using standard magnetohydrodynamical (MHD) approaches. Their strong surface fields $\left(B_{\text {pole }} \sim 10,000 \mathrm{kG}\right)$, paired with radiatively driven winds having modest mass-loss rates $\left(\dot{M} \sim 10^{-9} \mathrm{M}_{\odot} \mathrm{yr}^{-1}\right)$, imply magnetic confinement parameters on the order of $\eta_{*} \sim 10^{5}-10^{7}$. This parameter characterizes the relative energy densities of the magnetic field and the wind outflow, with the field dominating the wind in the limit $\eta_{*} \gg 1$, and vice versa in the limit $\eta_{*} \ll 1$ (for details, see ud-Doula \& Owocki 2002). In the large- $\eta_{*}$ limit, the field lines are almost rigid, and the Alfvén speed is very large; this makes MHD simulations very computationally expensive. (Asif ud-Doula's contribution above reports on simulations at the current upper limit of feasibility, $\eta_{*}=1,000$; this is still orders-of-magnitude smaller than the He-strong domain).

To tackle the strong-field limit, Townsend \& Owocki (2005) employed the simplifying Ansatz that the field lines are completely rigid; together with the frozen flux condition of ideal MHD, the result is that the field lines behave simply as fixed conduits (in the co-rotating frame of the star) for the wind material flowing along them. For closed field lines, it is inevitable that the wind streams from opposing footpoints will collide with each other, producing strong shocks in the manner envisaged by Babel \& Montmerle $(1997 \mathrm{a}, 1997 \mathrm{~b})$. As it cools down by radiative emission, the post-shock wind material is acted on by the gravitational and centrifugal forces. Beyond the Kepler co-rotation radius, the centrifugal force is sufficient to overcome gravity, and supports the cooled material near the tops of the rigid field loops in a warped, thin, co-rotating disk.

This Rigidly Rotating Magnetosphere model (RRM; Townsend \& Owocki 2005) has proven very successful in modeling the optical variability of the archetypal $\sigma$ Ori E (Townsend et al. 2005). However, the RRM model does not encompass the wind streams or shocks feeding the rigid magnetosphere, and therefore cannot be used for analysis of, e.g., X-ray emission. To incorporate these phenomena, we have developed a new RigidField Hydrodynamics (RFHD) approach that models the wind flow along each field line using a 1-D hydrodynamical code. By piecing together many independent 1-D models, we can build up a 3-D dynamical simulation for a complete magnetosphere in the strong-field limit, at a tiny fraction of the cost of an equivalent MHD simulation. Initial results from applying the RFHD approach to $\sigma$ Ori E confirm the analytical findings of the RRM formalism, but a number of novel results have also emerged. For instance, downstream of the shocks, the post-shock material is heated by centrifugal compression, to temperatures $\sim 10^{8} \mathrm{~K}$ where it emits hard X-rays; moreover, instabilities in the radiative cooling process excite periodic, dipole-mode oscillations in the disk material. Full details can be found in Townsend, Owocki \& ud-Doula (2007). (Acknowledgement: NASA/LTSA grant NNG05GC36G).

\section{Radiation Magneto-Hydrodynamics Simulations of Core-Collapse Supernovae}

Luc Dessart

Recent stellar evolutionary calculations of low-metalicity massive fast-rotating mainsequence stars yield iron cores at collapse that are endowed with high angular momentum. It is thought that high angular momentum and black hole $(\mathrm{BH})$ formation are critical ingredients of the collapsar model (Woosley 1993) of long-soft $\gamma$-ray bursts (GRBs). We recently performed (Dessart et al. 2008) 2D multi-group, flux-limited-diffusion MHD simulations of the collapse, bounce, and immediate post-bounce phases of a $35 M_{\odot}$ collapsarcandidate model of Woosley \& Heger (2006). We find that, provided the magnetorotational instability (MRI) operates in the differentially-rotating surface layers of the 
millisecond-period neutron star, a magnetically-driven explosion ensues during the protoneutron star phase, in the form of a baryon-loaded non-relativistic jet, and that a $\mathrm{BH}$, central to the collapsar model, does not form. Paradoxically, and although much uncertainty surrounds stellar mass loss, angular momentum transport, magnetic fields, and the MRI, current models of chemically homogeneous evolution at low metalicity yield massive stars with iron cores that may have too much angular momentum to avoid a magnetically-driven, hypernova-like, explosion in the immediate post-bounce phase. We surmise that fast rotation in the iron core may inhibit, rather than enable, collapsar formation, which requires a large angular momentum not in the core but above it.

We conclude that variations in the angular momentum distribution of pre-collapse massive stars may lead to different post-bounce scenarios. Non- or slowly-rotating progenitors may explode with weak/moderate energy $\left(\lesssim 1 \mathrm{~B} ; 10^{51} \mathrm{erg} \equiv 1\right.$ Bethe $\left.[1 \mathrm{~B}]\right)$ through a neutrino or an acoustic mechanism $\lesssim 1 \mathrm{~s}$ after bounce, or may collapse to a BH. Objects with large angular momentum in the envelope, but little in the core, may proceed through the proto-neutron star (PNS) phase, transition to a BH and form a collapsar with a GRB signature. Owing to the modest magnetic-field amplification above the PNS, a weak precursor polar jet may be launched, soon overtaken by a baryon-free, collimated relativistic jet. At the same time, the progenitor envelope is exploded by a disk wind, resulting in a hypernova-like SN with a large luminosity (large ${ }^{56} \mathrm{Ni}$ mass). Finally, and this is what we conclude here, objects with large angular momentum in the core may not transition to a BH. Instead, and fueled by core-rotation energy, a magnetically-driven baryon-loaded non-relativistic jet is obtained without any GRB signature. The explosion has the potential of reaching energies of a few $\mathrm{B}$ to $10 \mathrm{~B}$, and for viewers along the poles of looking like a Type Ic hypernova-like SN with broad lines. For a viewer at lower latitudes, the delayed and less energetic explosion nearer the equator may look more like a standard Type Ic SN (Höflich et al. 1999). This volume-restricted jet-like explosion is dimmer, as the amount of processed ${ }^{56} \mathrm{Ni}$ may be significantly less than the $\sim 0.5 M_{\odot}$ obtained in the collapsar context (MacFadyen \& Woosley 1999). Hence, magnetic processes during the post-bounce phase of fast-rotating iron cores offer a potential alternative to collapsar formation and long-soft GRBs by producing non-relativistic non-Poynting-flux-dominated baryon-loaded hypernova-like explosions without any GRB signature. Importantly, while our study narrows the range over which the collapsar model may exist, it also offers additional routes to explain the existence of GRB/SN-hypernova events like SN 1998bw (Woosley et al. 1999), and hypernova events like SN 2002ap without a GRB signature (Mazzali et al. 2002).

\section{References}

Babel, J. \& Montmerle, T. 1997a, A\&\&A, 323, 121

Babel, J. \& Montmerle, T. 1997b, ApJ, 485, L29

Dessart, L., Burrows, A., Livne, E., \& Ott, C. 2007, ApJ, 673, L43

Donati, J.-F., Semel, M., Carter, B. D., et al. 1997, MNRAS, 291, 658

Donati, J.-F., Paletou, F., Bouvier, J., \& Ferreira, J. 2005, Nature, 438, 466

Ferrario, L. \& Wickramasinghe, D. 2006, MNRAS, 367, 1323

Gagné, M., Oksala, M., Cohen, D. H., et al. 2005, ApJ, 628, 986

Höflich, P., Wheeler, J. C., \& Wang, L. 1999, ApJ, 521, 179

Hubrig, S., Briquet, M., Schöller, M., et al. 2006a, MNRAS, 369, L61

Hubrig, S., Yudin, R. V., Schöller, M., \& Pogodin, M. A. 2006b, A\&AA, 446, 1089

Hubrig, S., North, P., \& Schöller, M. 2007a, AN, 328, 475

Hubrig, S., Pogodin, M. A., Yudin, R. V., et al. 2007b, A\&SA, 463, 1039 
Hubrig, S., Schöller M., Briquet, M. et al. 2008, in: CP\#AP Workshop, Contrib. Astron. Obs. Skalnaté Pleso (arXiv:0712.0191)

Ignace, R. \& Gayley, K. G. 2007, arXiv:0708.1942

MacFadyen, A. I., \& Woosley, S. E. 1999, ApJ, 524, 262

Mazzali, P. A., Deng, J., Maeda, K. et al. 2002, ApJ, 572, L61

Shore, S. N. \& Brown, D. N. 1990, ApJ, 365, 665

Stelzer, B., Flaccomio, E., Montmerle, T., et al. 2005, ApJS, 160, 557

Townsend, R. H. D. \& Owocki, S. P. 2005, MNRAS, 357, 251

Townsend, R. H. D., Owocki, S. P., \& Groote, D. 2005, ApJ, 630, L81

Townsend, R. H. D., Owocki, S. P., \& Ud-Doula, A. 2007, MNRAS, 382, 139

ud-Doula, A. \& Owocki, S. P. 2002, ApJ, 576, 413

Wade, G. A., Fullerton, A. W., Donati, J.-F., et al. 2006, A\&SA, 451, 195

Weber, E. J. \& Davis, L. J. 1967, ApJ, 148, 217

Wojdowski, P. \& Schulz, N. S. 2005, ApJ, 627, 953

Woosley, S. E. 1993, ApJ, 405, 273

Woosley, S. E., Eastman, R. G., \& Schmidt, B. P. 1999, ApJ, 516, 788

Woosley, S. E. \& Heger, A. 2006, ApJ, 637, 914 